\title{
COLLECTIVE EXCITATIONS IN HEISENBERG ULTRATHIN FILMS WITH INHOMOGENEOUS SPATIAL DISTRIBUTION OF MAGNETISATION
}

\author{
R. EOSIŃSKI* AND Z. ONYSZKIEWICZ \\ Magnetism Theory Division, Faculty of Physics \\ A. Mickiewicz University, Umultowska 85, 61-614 Poznań, Poland \\ (Received July 7, 2000; in final form August 28, 2000)
}

The influence of non-magnetic substrate on the collective excitation of the Heisenberg $(s=1 / 2)$ ferromagnetic ultrathin films with homogeneous and inhomogeneous spatial distribution of magnetization is investigated. It has been proved that the collective excitation spectrum of ultrathin film may, to a significant degree, depend on the value of the coefficient characterizing the film coupling with its substrate.

PACS numbers: $75.10 . \mathrm{Jm}, 75.70 .-\mathrm{i}$

\section{Introduction}

Usually, in theoretical investigation of macroscopic bulk systems we do not have to take into account their interaction with the environment regarding the short-range character of intermolecular interactions. An effective interaction among the molecules of the system and its environment can only occur through the atoms on the system's surface or near it. The number of interacting atoms usually makes an insignificant part of all atoms of the system. However, considering ultrathin solid films and nanoscopic systems, the number of atoms interacting with the environment is frequently of the same order of magnitude as the total number of atoms in the system. Moreover, thin solid films are deposited on bulk substrates whose structure influences that of the films. Because of that we have to take into account the interaction between the substrate and the film. Usually, in order to do that we introduce phenomenological surface parameters. The values of these parameters significantly influence the physical properties of thin solid films which is for example evidenced in their spectra of collective excitations.

*corresponding author, e-mail: rlosin@main.amu.edu.pl 
In this paper we propose a general method enabling to take into account the interaction between the solid film and the substrate on a microscopic level. In the method proposed this interaction is included already in construction of the reduced density operator [1-3].

The aim of our paper is to give a detailed analysis of the influence of non-magnetic substrate of the collective excitations for the Heisenberg $(s=1 / 2)$ ferromagnetic ultrathin films with homogeneous and inhomogeneous spatial distribution of magnetization.

\section{Collective excitations spectrum} $[1,2]$

Taking advantage of the generalised equilibrium reduced density operator

$$
d=\exp \left[\beta\left(F-H-H^{\prime}\right)\right]
$$

where

$$
\begin{aligned}
& H^{\prime}=\operatorname{Tr}_{s}\left[H_{\mathrm{I}} d_{s}\right], \\
& F=-\frac{1}{\beta} \ln \operatorname{Tr}\left\{\exp \left[-\beta\left(H+H^{\prime}\right)\right]\right\}
\end{aligned}
$$

is the free energy of the ultrathin film, $d_{s}$ - equilibrium reduced density operator of the substrate, $\beta=1 / k_{\mathrm{B}} T$ and

$$
\begin{aligned}
H= & -\frac{1}{2} \sum_{\boldsymbol{f} \neq \boldsymbol{f}^{\prime}} \sum_{r} \sum_{\alpha, \gamma} K_{\boldsymbol{f} f^{\prime} r}^{\alpha \gamma} S_{\boldsymbol{f} r}^{\alpha} S_{\boldsymbol{f}^{\prime} r}^{\gamma}-\frac{1}{2} \sum_{\boldsymbol{f}, r} \sum_{\alpha, \gamma} K_{f r, r+1}^{\alpha \gamma} S_{\boldsymbol{f} r}^{\alpha} S_{\boldsymbol{f} r+1}^{\gamma} \\
& -\frac{1}{2} \sum_{\boldsymbol{f}, r} \sum_{\alpha, \gamma} K_{\boldsymbol{f} r, r-1}^{\alpha \gamma} S_{\boldsymbol{f} r}^{\alpha} S_{\boldsymbol{f} r-1}^{\gamma}+\frac{1}{2} C \varepsilon^{2}
\end{aligned}
$$

where $\alpha \gamma$ are equal to,+--+ or $z z$, and

$$
\begin{aligned}
& S^{ \pm}=S^{x} \pm \mathrm{i} S^{y}, \\
& K_{f f^{\prime} r}^{\alpha \gamma}=K_{f r, r \pm 1}^{\alpha \gamma}= \begin{cases}\frac{1}{2} I & \text { for } \alpha \gamma=+- \text { or }-+ \\
I & \text { for } \alpha \gamma=z z,\end{cases}
\end{aligned}
$$

$\operatorname{Tr}_{s}[\ldots]$ is the partial trace over the state of the substrate.

Here $K_{f f^{\prime} r}^{\alpha \gamma}$ is the intra-monoatomic layer interaction coefficient, $K_{f r, r \pm 1}^{\alpha \gamma}$ is the inter-monoatomic layer ferromagnetic interaction and $f$ denotes the bidimensional position vectors of spins belonging to a given monoatomic layer. The summation runs always over different sites. According to the Valenta [4] model, the film is divided into $R$ monoatomic layers parallel to the planes (100) of a simple cubic lattice. The position of each monoatomic layer is defined by the number $r=1,2, \ldots, R$. Our considerations are restricted to the nearest neighbour interactions only. The relevant component of the film strain tensor is denoted as $\varepsilon$. The specific properties of thin film are related to the physical fact of the lack of neighbours of spins in the boundary layers and the interaction with the substrate described by $H_{\mathrm{I}}$. 
We assume that the atoms on the surface of the non-magnetic substrate of the thin film affect the shape of the electronic clouds of the atoms of the film made of a monoatomic layer $r=1$. This in turn modifies the exchange interactions coupling the spins from the monoatomic layer $r=1$. We also assume that this modification depends on the positions of the substrate atoms on its surface which are in contact with the layer. The positions of these atoms will be described by a position vector $g$. Therefore, the interaction of the layer with the substrate, made of one kind of atoms not endowed with a magnetic moment, can be described as

$$
H_{\mathrm{I}}=-\frac{1}{2} \sum_{\boldsymbol{f} \neq \boldsymbol{f}^{\prime}, r=1} \sum_{\alpha \gamma} \sum_{\boldsymbol{g}} D^{\alpha \gamma}\left(\boldsymbol{f}-\boldsymbol{g}, \boldsymbol{f}^{\prime}-\boldsymbol{g}\right) S_{\boldsymbol{f} r}^{\alpha} S_{\boldsymbol{f}^{\prime} r}^{\gamma},
$$

where $D^{\alpha \gamma}\left(f-g, f^{\prime}-g\right)$ is a fast decreasing function of the variables $|f-g|$ and $\left|f^{\prime}-g\right|$, defining the distances between the atoms of the layer and those of the substrate. The symbol $\sum_{g}$ stands for summation over all atoms of the substrate in contact with the layer $r=1$. The specific form of this function depends on the details of the microstructure of the contact between the layer and the substrate and the kind of atoms making them.

On substituting (6) into (2), we get

$$
H^{\prime}=\frac{1}{2} \sum_{f \neq f^{\prime}, r=1} \sum_{\alpha \gamma} D_{f f^{\prime}}^{\alpha \gamma} S_{f}, \alpha S_{f^{\prime} r},
$$

where

$$
D_{f f^{\prime}}^{\alpha \gamma}=\operatorname{Tr}_{s}\left[\sum_{g} D^{\alpha \gamma}\left(f-g, f^{\prime}-g\right) d_{s}\right] .
$$

Not knowing the explicit form of $D_{f f^{\prime}}^{\alpha \gamma}$ and its temperature dependence, we assume that it is proportional to the deformation of the monoatomic layer $r=1$, described by the relevant component of the film strain tensor $\varepsilon$. Thus, we assume that

$$
D_{f f^{\prime}}^{\alpha \gamma}= \begin{cases}\frac{1}{2} I_{1} \varepsilon & \text { for } \alpha \gamma=+- \text { or }-+, \\ I_{1} \varepsilon & \text { for } \alpha \gamma=z z\end{cases}
$$

where $I_{1}$ is a proportionality constant being the model parameter describing the intensity of the interaction between the monoatomic layer $r=1$ and the substrate. Therefore, $H^{\prime}$ depends on temperature through the following dependence: $\varepsilon=\varepsilon(\beta)$.

$$
\begin{aligned}
& \text { Thus, } \\
& \bar{H}=H+H^{\prime}
\end{aligned}
$$

is the effective Hamiltonian describing the ultrathin film interacting with the substrate.

In order to consider the collective excitations in model (10), we have to introduce a causal matrix two-spin Green function depending on temperature, applied to the transverse components of spin operators [5]:

$$
\breve{G}=\left\{G_{r r^{\prime}}\right\},
$$

where

$$
G_{r r^{\prime}}=\left\langle\hat{T} \tilde{S}_{\boldsymbol{f} r}^{-}(\tau) \tilde{S}_{\boldsymbol{f}^{\prime} r^{\prime}}^{+}(0)\right\rangle,
$$




$$
\langle\ldots\rangle=\operatorname{Tr}[\ldots d]
$$

and $d$ is given by Eq. (1). The components of spin operators appear here in the "Heisenberg" representation,

$$
\tilde{S}^{ \pm}(\tau)=\exp (\bar{H} \tau) S^{ \pm} \exp (-\bar{H} \tau)
$$

and the parameter $\tau$ assumes values within the range $0<\tau<1 / k_{\mathrm{B}} T$.

The Green function $\breve{G}$ is defined in the above restricted range and therefore, it can be developed into a Fourier series with respect to discrete frequencies $\omega_{n}$

$$
G_{r r^{\prime}}=\left(f-f^{\prime}, \tau\right)=\frac{1}{N} \sum_{k} \sum_{n} \exp \left\{\mathrm{i}\left[\omega_{n} \tau-k \cdot\left(f-f^{\prime}\right)\right]\right\} G_{r r^{\prime}}\left(k, i \omega_{n}\right),
$$

where $n=0, \pm 1, \pm 2, \ldots$ and $k$ is planar wave vector, $\omega_{n}=2 \pi \beta^{-1} n$ whereas $N$ number of spins in one monoatomic layer.

In order to find the matrix Green function $\breve{G}$ we will use the renormalised high-density expansion method [5]. The essential part of this method is the renormalisation of the line of the interactions between the transverse spin components, performed employing a correlation function which includes Gaussian fluctuations of molecular field. This renormalisation yields better results than random phase approximation (RPA), especially in the vicinity of the critical points, where strong molecular field fluctuations play an important role. Another advantage of this method is the fact that its results are valid in a wide temperature range.

As a starting point to renormalise the high-density expansion, we choose the following decomposition of the effective Hamiltonian (10):

$$
\bar{H}=\left(\bar{H}-\bar{H}_{1}\right)+\bar{H}_{1}=\bar{H}_{0}+\bar{H}_{1},
$$

where the perturbative part $\bar{H}_{1}$ is defined by the transformation:

$$
\bar{H} \rightarrow \bar{H}_{1}=\bar{H}\left(S^{z} \rightarrow \delta S^{z}\right)
$$

and

$$
\delta S_{\boldsymbol{f} r}^{z}=S_{\boldsymbol{f} r}^{z}-\left\langle S_{r}^{z}\right\rangle
$$

is the fluctuation operator of the $z$-component of the spin, where

$$
\left\langle S_{r}^{z}\right\rangle=\operatorname{Tr}\left[S_{f r}^{z} d\right] \text {. }
$$

The Hamiltonian $\bar{H}_{0}$ is equivalent to $\bar{H}$ written in the molecular field approximation (MFA). The Green function can be expanded into a series with respect to the perturbing term $\bar{H}_{1}$.

From among the Feynman diagrams contributing corrections to the Fourier transform of the matrix Green function, we can distinguish the ones which cannot be cut by means of cutting a single interaction line. These diagrams will be referred to as the irreducible part of the matrix Green function and are denoted as $\breve{\Sigma}$.

The Fourier transform of the matrix Green function

$$
\breve{G}\left(k, i \omega_{n}\right)=\left\{G_{r r}\left(k, i \omega_{n}\right)\right\}
$$

and the irreducible part $\breve{\Sigma}$ satisfy the following matrix equation (i.e. Larkin equation [6] similar to the Dyson equation in the quantum field theory):

$$
\breve{G}\left(k, \mathrm{i} \omega_{n}\right)=\breve{\Sigma}\left(k, \mathrm{i} \omega_{n}\right)+\breve{\Sigma}\left(k, \mathrm{i} \omega_{n}\right) \breve{K}^{-+}(k) \breve{G}\left(k, i \omega_{n}\right),
$$


which expresses certain topological features of their diagrams, where

$$
\breve{K}^{-+}(k)=\beta\left\{K_{r r^{\prime}}^{-+}(k)\right\}
$$

is the matrix Fourier transform of the interaction coefficients, and

$$
K_{r r^{\prime}}^{-+}(k)=\frac{1}{N} \sum_{f-f^{\prime}} K_{r r^{\prime}}^{-+}\left(f-f^{\prime}\right) \exp \left[\mathrm{i} k \cdot\left(f-f^{\prime}\right)\right] .
$$

Applying a matrix notation, the solution of Eq. (21) can be written in a compact form

$$
\breve{G}\left(k, \mathrm{i} \omega_{n}\right)=\left[\breve{1}-\breve{\Sigma}\left(k, \mathrm{i} \omega_{n}\right) \breve{K}^{-+}\left(k, \mathrm{i} \omega_{n}\right)\right]^{-1} \breve{\Sigma}\left(k, \mathrm{i} \omega_{n}\right) .
$$

Considering the structure of Eq. (24) we can find that the poles of the Fourier transform of the matrix Green function are given by

$$
\operatorname{det}\left[\breve{I}-\breve{\Sigma}\left(k, \mathrm{i} \omega_{n}\right) \breve{K}^{-+}(k)\right]=0 .
$$

After analytical extension

$$
\mathrm{i} \omega_{n} \rightarrow \omega+\mathrm{i} \delta \operatorname{sgn} \omega \quad(\delta \rightarrow+0)
$$

the $\breve{G}\left(k, i \omega_{n}\right)$ becomes equivalent to a Fourier transform of the matrix causal time Green function poles which determine a spectrum of collective excitations of a system [7] (in our case ultrathin film).

Thus, it is evident that the determination of collective excitations in an ultrathin film boils down to finding the irreducible part of the matrix Green function. We found this irreducible part $\breve{\Sigma}\left(k, i \omega_{n}\right)$ with the help of the high-density expansion scheme in the zero approximation with respect of the interactions of transverse spin coordinates, being renormalized by the correlation functions of operators of longitudinal spin coordinates fluctuations. In the theory of the spin system, high-density expansion scheme is based on the classification of the Feynman diagrams with respect to powers of the parameter $1 / z$, where $z$ is the effective number of spins interacting with any given spin $[6,8,9]$. The approach is valid when $z \gg 1$.

The correlation functions mentioned are calculated in the approximation including Gaussian fluctuations of the molecular field, like in [5, 10], which is a modified version of the high-density expansion method. The Gaussian fluctuations approximation (GFA) is an improvement over the MFA as it allows the self-consistent inclusion of Gaussian fluctuations of this field. The essential new element of GFA is the summation of the partial sums of Feynman diagrams of the structure of recurrent formula at each stage of the calculations. Owing to this procedure, the theory becomes internally consistent and does not lead to non-physical results, such as e.g. a complex Curie temperature (see Ref. [11]). In GFA for matrix $\breve{\Sigma}\left(k, i \omega_{n}\right)$ we have chosen a partial sum which can be represented graphically as 


$$
\breve{\Sigma}\left(k, i \omega_{n}\right)=\left|\begin{array}{cccc}
\longrightarrow \bullet & 0 & 0 & \ldots \\
0 & \longrightarrow \bullet & 0 & \ldots \\
0 & 0 & \bullet_{33} & \cdots \\
\ldots & \ldots & \ldots & \ldots
\end{array}\right|_{R \times R}
$$

where

$$
\rightarrow \bullet \longrightarrow_{r r}=\longrightarrow 0-0{ }_{r r}+\longrightarrow 000-0
$$

is the high-density expansion and the symbols - denote the renormalised interaction line,

$$
\begin{aligned}
& { }_{r}{ }_{r}=\cdots \cdots 0 \bullet 0 \cdots{ }_{r r}+\cdots+\cdots 0 \bullet 0 \cdots{ }_{r-1, r-1} \\
& +\cdots \cdots \circ \bullet \circ \cdots \cdots_{r+1, r+1} \\
& \underbrace{\circ \circ \cdots \circ}_{m}=\frac{\partial^{m}}{\partial y_{r}^{m}} L\left(y_{r}\right) \text {, } \\
& { }_{r} \cdots r_{r}=\beta I(k)
\end{aligned}
$$

is the high-density expansion. Hence

$$
\longrightarrow \bullet{ }_{r r}=G_{r}\left(\mathrm{i} \omega_{n}\right) m_{r}
$$

and

$$
G_{r}\left(i \omega_{n}\right)=\left(y_{r}-\mathrm{i} \omega_{n} \beta\right)^{-1}
$$

where molecular field in the $r$-th monoatomic layer parallel to surfaces of the ultrathin films is

$$
\begin{aligned}
& y_{r}=\frac{1}{t}\left[m_{r}\left(1+\delta_{r, 1} I_{p} \varepsilon\right)+\frac{1}{4}\left(1-\delta_{r, 1}\right) m_{r-1}+\frac{1}{4}\left(1-\delta_{r, R}\right) m_{r+1}\right] \\
& t=(4 I \beta)^{-1} \\
& I_{p}=I_{1}(I)^{-1} .
\end{aligned}
$$

Moreover, the magnetization

$$
m_{r}=\frac{1}{\sqrt{2 \pi}} \int_{-\infty}^{+\infty} \exp \left(-\frac{1}{2} x^{2}\right) \frac{\partial}{\partial y_{r}} L\left(y_{r}+x \delta y_{r}\right) \mathrm{d} x .
$$

Mean Gaussian fluctuation $\delta y_{r}$ of the molecular field $y_{r}$ is given by the formula

$$
\begin{aligned}
\delta y_{r} & =\frac{1}{8 t}\left\{\frac { 1 } { \sqrt { 2 \pi } } \int _ { - \infty } ^ { + \infty } \operatorname { e x p } ( - \frac { 1 } { 2 } x ^ { 2 } ) \left[4 \frac{\partial^{2}}{\partial y_{r}^{2}} L\left(y_{r}+x \delta y_{r}\right)\right.\right. \\
& +\left(1-\delta_{r, 1}\right) \frac{\partial^{2}}{\partial y_{r-1}^{2}} L\left(y_{r-1}+x \delta y_{r-1}\right) \\
& \left.\left.+\left(1-\delta_{r, R}\right) \frac{\partial^{2}}{\partial y_{r+1}^{2}} L\left(y_{r+1}+x \delta y_{r+1}\right)\right] \mathrm{d} x\right\}^{1 / 2},
\end{aligned}
$$


and the relevant component of the film strain tensor $\varepsilon$ satisfies the stationary condition

$$
\frac{\partial F}{\partial \varepsilon}=0
$$

Hence

$$
\varepsilon=\frac{4 t N I}{C \sqrt{2 \pi}} \int_{-\infty}^{+\infty} \exp \left(-\frac{1}{2} x^{2}\right) \frac{\partial}{\partial \varepsilon} L\left(y_{1}+x \delta y_{1}\right) \mathrm{d} x,
$$

where

$$
L\left(y_{r}+x \delta y_{r}\right)=\ln \left\{2 \cosh \left[\frac{1}{2}\left(y_{r}+x \delta y_{r}\right)\right]\right\} .
$$

After analytical extension, poles (25) determine a spectrum of collective excitations of our model.

\section{Results}

For the ferromagnetic ultrathin film with a homogeneous distribution of magnetisation $m$ defined as

$$
m=\frac{1}{R} \sum_{r=1}^{R} m_{r}
$$

and an non-homogeneous spatial distribution of magnetisation $m_{r}$, for the planar
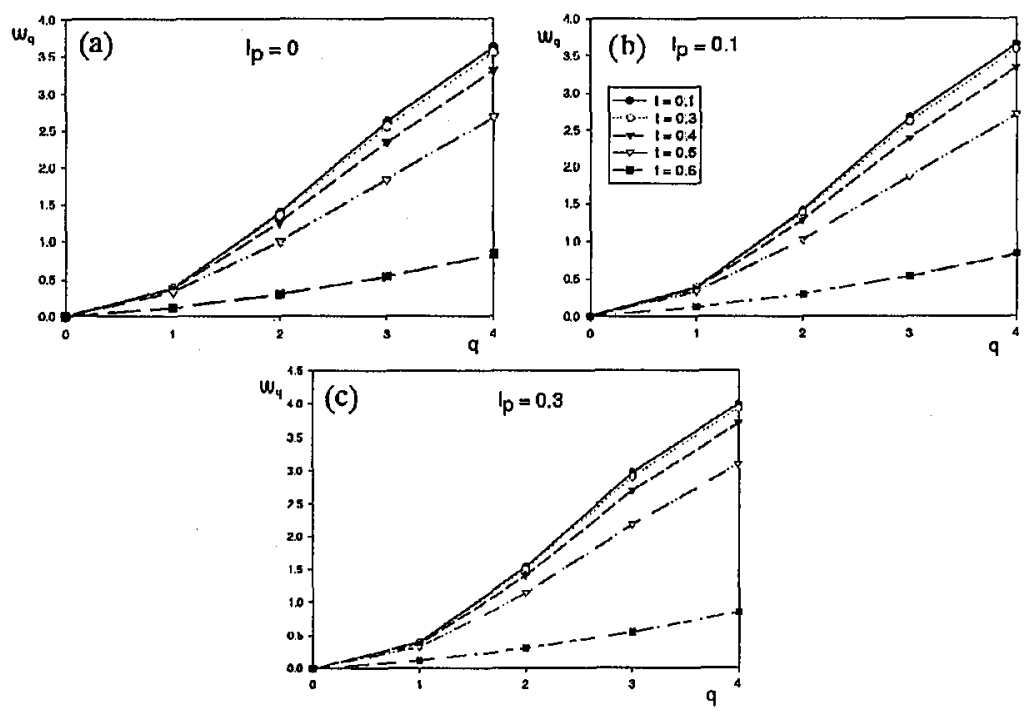

Fig. 1. Collective excitation spectrum (in relative units) of an ultrathin film for different temperatures $t=(4 I \beta)^{-1}$ and $I_{p}=I_{1} / I\left(k=0, R=5\right.$ for (a) $I_{p}=0$, (b) $I_{p}=0.1$ and (c) $\left.I_{p}=0.3\right)$. 

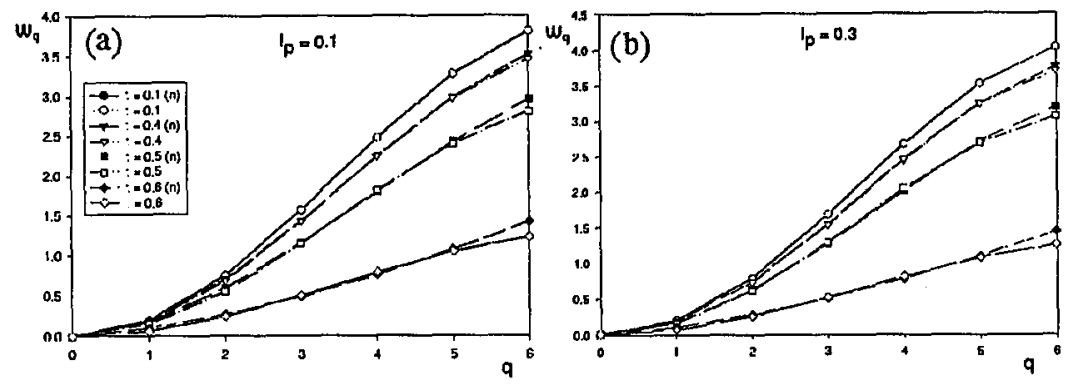

Fig. 2. Collective excitation spectrum of an ultrathin film (in relative units) for different temperatures $t=(4 I \beta)^{-1}$ with homogeneous and non-homogeneous (n) spatial distribution of the magnetization ( $\mathbb{k}=0, R=7$ for (a) $I_{p}=0.1$ and (b) $I_{p}=0.3$ ).

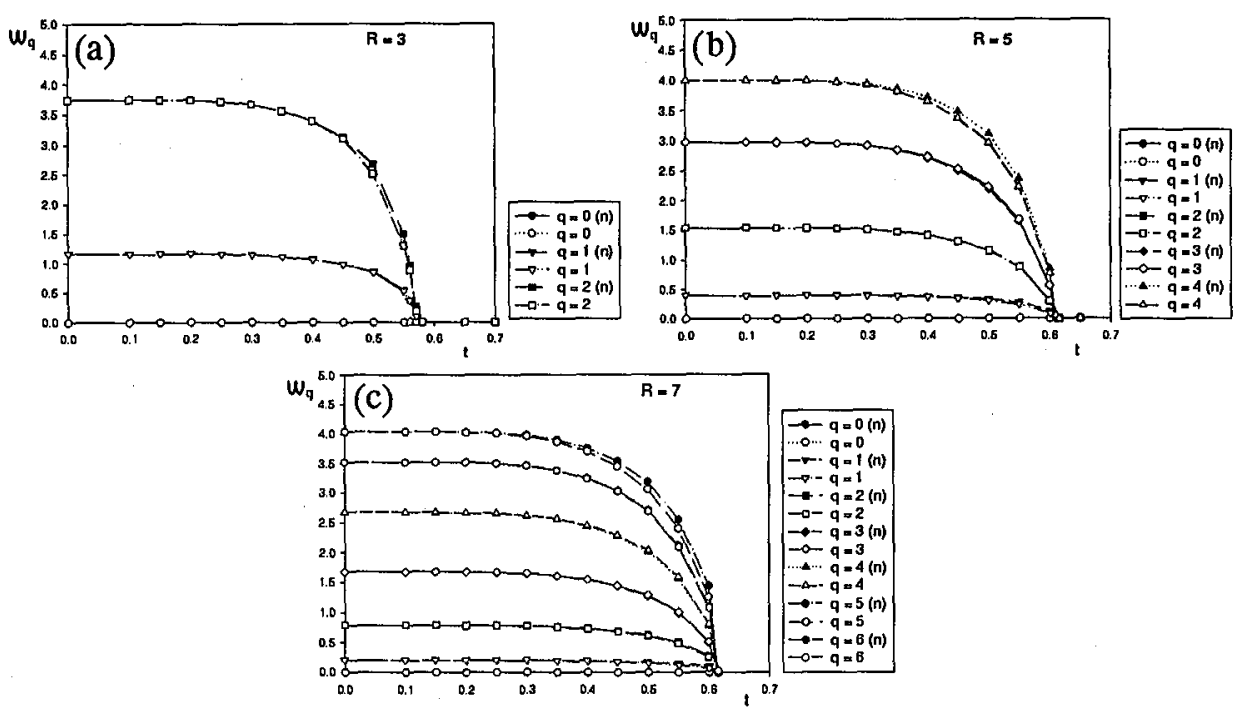

Fig. 3. Collective excitation frequency $\omega_{q}$ (in relative units) against temperature $t=(4 I \beta)^{-1}$ for ultrathin film with homogeneous and non-homogeneous (n) spatial distribution of the magnetization ( $k=0$ for (a) $R=3$, (b) $R=5$ and (c) $R=7$ ).

wave vector $k=0$, the collective excitation spectra in the direction perpendicular to the surface of the film were calculated from Eq. (25) in the whole temperature range considered. Equations (25)-(40) are solved numerically for second-order elastic moduli of the film $C / 4 N I=1$ and different: numbers of monoatomic layers $R$, temperatures $t=(4 I \beta)^{-1}$ and coefficient $I_{p}=I_{1} / I$ characterizing the film coupling with its substrate. We obtain $R$ solutions, ordered by $q=0,1,2, \ldots, R-1$. They correspond to the poles of the Green function interpreted as collective excitation frequency $\omega_{q}$ propagating in the direction perpendicular to surface of the ultrathin ferromagnetic film. Therefore, $q$ can be considered as a component of the wave vector perpendicular to the film surface. As only the collective excitations 
propagating in perpendicular to the film surface depend on the spatial distribution of magnetisation, in numerical calculations we assume that the wave vector components parallel to the film surface are equal to zero $k=0$. The results are presented graphically.

Figure 1 presents the collective excitation spectrum of the ferromagnetic ultrathin film for different temperatures $t$ and different $I_{p}(k=0, R=5)$. The collective excitation spectrum of the ultrathin film for different temperatures $t$ with a homogeneous and non-homogeneous (n) spatial distribution of the magnetisation ( $k=\mathbf{0}, R=7)$ is shown in Fig. 2. Then in Fig. 3 we show the collective excitation frequency $\omega_{q}$ against temperature $t$ for the films with homogeneous and non-homogeneous (n) spatial distribution of the magnetisation for $R=3,5$, and 7 $(k=0)$.

\section{Conclusion}

In conclusion, we can say that the results presented in Figs. 1 to 3 , prove that the collective excitation spectrum in direction perpendicular to surface of the film of ultrathin ferromagnetic films may, to a significant degree, depend on the value of the $I_{p}$ coefficient characterizing the film coupling with its substrate. Moreover, the results obtained show that the collective excitation spectrum of the ferromagnetic ultrathin films weakly depends on inhomogeneity of the spatial distribution of magnetization $m_{r}$. As seen from Fig. 2, the inhomogeneity of the spatial distribution of magnetization in the ultrathin film leads to a more linear shape than that predicted by the relation $\omega_{q} \propto q^{2}$. This result is in a full agreement with that obtained by the method of double-time statistical Green function in the Tyablikov decoupling approximation reported in [12].

In the work [12] within the Tyablikov approximation, the inhomogeneities of the spatial distribution of magnetisation were taken into account only in an approximate manner, in the first order of a relevant perturbation calculus, in which the deviation of magnetisation from the uniform distribution was assumed as a small parameter. In this work, within the GFA, the influence of he inhomogeneity of the magnetisation distribution on $\omega_{q}$ has been taken into account in the exact way. Moreover, a new point of this work has been the investigation of the effect of the substrate on the spectrum of collective excitations for an ultrathin film. The method used in this work can be successfully used for investigation of collective excitations and their substrate dependence for nanoscopic magnetic particles. The results are expected to be of interest for the nanophysics of magnetic materials.

This work is supported by the Committee for Scientific Research under grant No. 2P03B075 14.

\section{References}

[1] Z. Onyszkiewicz, Phys. Status Solidi B 150, 109 (1988).

[2] Z. Onyszkiewicz, Phys. Lett. A 163, 450 (1992).

[3] Z. Jacyna-Onyszkiewicz, The Principles of Quantum Thermodynamics, A. Mickiewicz University Press, Poznań 1996 (in Polish). 
[4] L. Valenta, Czech. J. Phys. 7, 127 (1957).

[5] Z. Onyszkiewicz, Physica A 103, 274 (1980).

[6] Yu.A. Izyumov, F.A. Kassan-Ogly, Yu.N. Skryabin, Field Methods in the Theory of Ferromagnets, Mir, Moscow 1974 (in Russian).

[7] A.A. Abrikosov, L.P. Gorkov, I.E. Dzyaloshinsky, Method of Quantum Field Theory in Statistical Physics, Prentice-Hall, New York 1963.

[8] R. Brout, Phys. Rev. 118, 1009 (1960); 122, 469 (1961).

[9] F. Englert, Phys. Rev. 129, 567 (1963).

[10] Z. Onyszkiewicz, A. Wierzbicki, J. Magn. Magn. Mater. 99, 253 (1991).

[11] Z. Onyszkiewicz, A. Wierzbicki, Phys. Lett. A 116, 335 (1986).

[12] Z. Onyszkiewicz, Phys. Status Solidi 41, 161 (1970). 